\title{
IMPROVED EFFICIENCY IN LEGGED RUNNING USING LIGHTWEIGHT PASSIVE COMPLIANT FEET*
}

\author{
MARCO HUTTER, CLAUDE HOLENSTEIN, DARIO FENNER, C. DAVID REMY, \\ MARK A. HOEPFLINGER, and ROLAND SIEGWART \\ Autonomous Systems Lab, Institute of Robotics and Intelligent Systems, \\ Swiss Federal Institute of Technology (ETHZ), Zurich, Switzerland, mahutter@ethz.ch
}

\begin{abstract}
This paper investigates the mechanical benefits of employing a passive foot segment to improve energetic efficiency in legged running. The proposed lightweight design significantly reduces impact and damping losses, while simultaneously allowing for a natural-looking stance configuration. Actuator input and ankle spring properties were optimized in simulation and successfully tested in $2 \mathrm{D}$ running experiments.
\end{abstract}

Keywords: compliant leg design, passive ankle joint, efficient hopping, SEA

\section{Introduction}

Locomotion in nature is highly effective in terms of speed and energetic efficiency. To achieve both, elastic elements in muscles and tendons play a highly important role by introducing a passive support to the vertical oscillation of the main body, as it is found in all dynamic gaits of animals and humans [1]. Inspired by these principles, research has tried to transform the idea of elastic energy storage into robotic devices using series elastic actuation. Springs in each joint can not only be used as force elements to accurately control the joint torque, but also act as passive elements that enhance the natural dynamics of the system. The springs can store energy during the deceleration phase after landing and subsequently release it before lift-off. In the optimal case, the actuator must never produce negative work, but uses the entire stance phase to introduce energy into the system [2].

While optimal control strategies allow exploiting the natural dynamics in a very efficient manner [3], major limitations are given by the mechanical

*This research was supported by the Swiss National Science Foundation through the National Centre of Competence in Research Robotics. 

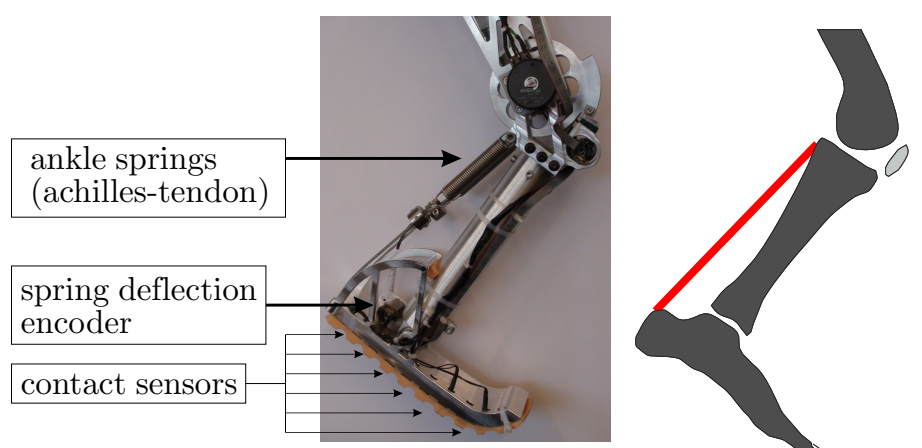

Fig. 1. The robotic foot is built similar to a human leg. Springs emulate the behavior of the Achilles tendon and Soleus muscle. An orthopedic foot sole ensures a soft impact, and sensors allow contact detection and precise joint torque measurement.

structure of the hardware system. For example, the capability to store energy depends heavily on the damping and friction effects in the joint springs. In addition to this, a second substantial drain of energy in dynamic maneuvers can be attributed to impact collision at landing. In articulated legs such as StarlETH [4], these energy losses largely depend on the configuration at impact and on the individual segment mass. Although swing leg retraction [5] or pre-impact speed adaptation potentially allow to lower these losses, their implementation in real robotic devices is often difficult to realize.

In this paper we describe the development and mechanical optimization of a passive foot element (Figure 1). This is done in theory, simulation, and on a real robotic system, with the overall goal of increasing the efficiency of one-legged running. Optimization strategies are not only used to improve motor control policies, but also to optimize the mechanical properties of the leg. Inspired by biology, the foot is equipped with springs emulating the behavior of the Achilles tendon and Soleus muscle (Figure 1), while, at the same time, the sole of an orthopedic foot absorbs the impact shocks.

\section{Impact Analysis of Segmented Legs}

The dynamics equation of a system colliding with its environment can be described by $\mathbf{M}\left(\dot{\mathbf{q}}^{+}-\dot{\mathbf{q}}^{-}\right)+\mathbf{J}_{s}^{T} \mathcal{F}_{s}=\mathbf{0}$ with the generalized velocities before $\left(\dot{\mathbf{q}}^{-}\right)$and after $\left(\dot{\mathbf{q}}^{+}\right)$impact, the mass matrix $\mathbf{M}$, the support Jacobian $\mathbf{J}_{s}$, and the contact impulsive force $\mathcal{F}_{s}$. Since the contact point is immediately brought to zero velocity $\dot{\mathbf{r}}_{s}^{+}=\mathbf{J}_{s} \dot{\mathbf{q}}^{+}=\mathbf{0}$, the instantaneous change in generalized velocities is given as

$$
\Delta \dot{\mathbf{q}}=\dot{\mathbf{q}}^{+}-\dot{\mathbf{q}}^{-}=-\mathbf{M}^{-1} \mathbf{J}_{s}^{T}\left(\mathbf{J}_{s} \mathbf{M}^{-1} \mathbf{J}_{s}^{T}\right)^{-1} \mathbf{J}_{s} \dot{\mathbf{q}}^{-}=-\overline{\mathbf{J}}_{s} \mathbf{J}_{s} \dot{\mathbf{q}}^{-},
$$




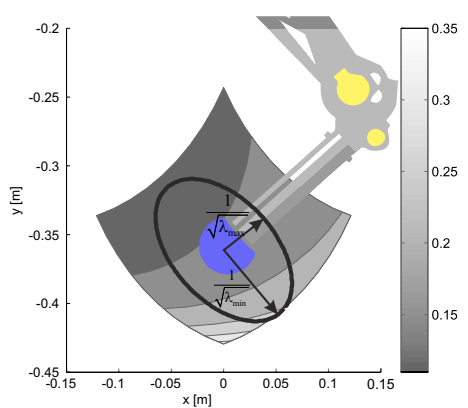

(a) GIE for ScarlETH

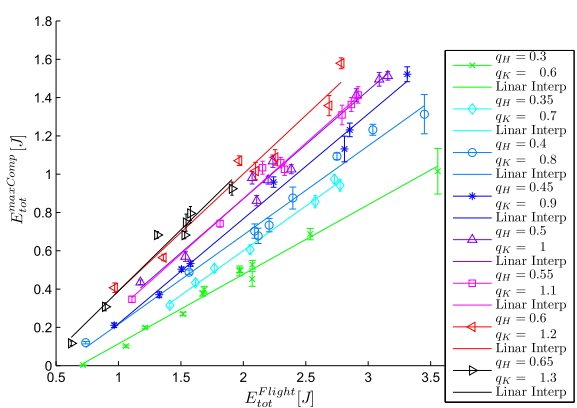

(b) Drop-Test energy loss

Fig. 2. A GIE analysis (b) indicates that the pre-impact velocity should be perpendicular to the shank in order to minimize the impact losses. Theoretic analysis (a) and experimental validations (b) show that a crouched position results in lower energy losses.

with the support point inertia $\boldsymbol{\Lambda}_{s}:=\left(\mathbf{J}_{s} \mathbf{M}^{-1} \mathbf{J}_{s}^{T}\right)^{-1}$ and the dynamically consistent generalized inverse of the support Jacobian $\overline{\mathbf{J}}_{s}=\mathbf{M}^{-1} \mathbf{J}_{s}^{T} \boldsymbol{\Lambda}_{s}[6]$. The energy loss in such a collision can be written as

$$
E_{\text {loss }}=\Delta E_{k i n}=-\frac{1}{2} \Delta \dot{\mathbf{q}}^{T} \mathbf{M} \Delta \dot{\mathbf{q}}
$$

This can be expressed in task space using $\Delta \dot{\mathbf{r}}_{s}=-\dot{\mathbf{r}}_{s}^{-}=\mathbf{J}_{s} \Delta \dot{\mathbf{q}}$ as

$$
E_{\text {loss }}=\Delta E_{k i n}=-\frac{1}{2} \Delta \dot{\mathbf{r}}_{s}^{T} \boldsymbol{\Lambda}_{s} \Delta \dot{\mathbf{r}}_{s}=-\frac{1}{2} \dot{\mathbf{r}}_{s}^{-T} \boldsymbol{\Lambda}_{s} \dot{\mathbf{r}}_{s}^{-} .
$$

Since only part of the derivation was given in [7], the entire proof for (2) and (3) is compactly outlined in (A.1) and (A.2).

The complex impact mechanics can be analyzed through an eigenvalue decomposition of the foot point inertia $\boldsymbol{\Lambda}_{s}$. The highest impact energy loss occurs if the pre-impact speed $\dot{\mathbf{r}}_{s}^{-}$is aligned with the eigenvector corresponding to the greatest eigenvalue. In [8] and [7] a visual interpretation is proposed by plotting the generalized inertia ellipsoid (GIE) with the perpendicular axis $1 / \sqrt{\lambda_{1,2}} \mathbf{e}_{1,2}$.

\section{Implications on a 2-Segmented Leg}

Investigation of different landing configurations for the 2-segmented leg ScarlETH $[2,4]$ based on the GIE (Figure 2(a)) indicates that impact energy losses are minimal if the pre-impact velocity $\dot{\mathbf{r}}_{s}^{-}$is nearly perpendicular to the inertia main axis of the contact segment. For in-place hopping, this implies that energy losses are minimized for a crouched positions, ideally with the shank segment being close to horizontal (dark area, $<15 \%$ energy loss). On the other hand, more elongated leg configurations (bright area, 
$>25 \%$ energy loss) have a higher end-effector inertia in vertical direction and hence significantly more energy drain with the same vertical pre-impact speed.

To confirm these theoretical results on an actual robotic device, we conducted several experiments with ScarlETH. In reality, a contact collision is not an instantaneous process. It induces extremely high accelerations at the different segments which is very difficult to capture in measurements. Additional losses due to friction or damping effects that are impossible to model require to evaluate efficiency in a different way. As a very intuitive solution, we propose to measure how much of the center of gravity (CoG) energy during flight phase

$$
E_{t o t}^{-}=m g\left(y_{C o G}-y_{0}\right)+\frac{1}{2} m \dot{\mathbf{r}}_{C o G}^{2}
$$

can be preserved until the mid-stance point. At that point, the velocity of all segments is zero and the knee spring (characteristic $c(\delta)$ identified in [4]) is maximally compressed

$$
E_{\max C o m p}=m g\left(y_{C o G}-y_{0}\right)+\int_{0}^{\delta_{\max }} c(\delta) d \delta .
$$

Since energy is constant during flight phase, all available measurements between apex (highest point in flight curve) and touchdown are used to estimate (4). The energy at maximal compression is accurately determinable as we do not have to account for kinetic energy that is prone to noise.

The results of multiple dropping tests are depicted in Figure 2(b). Taking the height relative to the leg length at impact ensures that only the actual jumping height contributes to the potential energy. For a constant landing configuration the dependency between the energy level (4) and the energy loss (3) is linear, with an increasing slope for less crouched configurations. This is in agreement with the theory: Since a different starting height changes only the norm of $\dot{\mathbf{r}}_{s}^{-}=\dot{\mathbf{r}}_{C o G}^{-}$but not its direction, (3) reduces to $-\frac{1}{2} \lambda\left(\dot{\mathbf{r}}_{s}^{-}\right)^{2}$ with the geometric scalar $\lambda$, and the relation $\frac{\Delta E_{\text {kin }}}{E_{\text {tot }}^{-}}=\frac{-1 / 2 \lambda\left(\dot{\mathbf{r}}_{s}^{-}\right)^{2}}{1 / 2 m\left(\dot{\mathbf{r}}_{s}^{-}\right)^{2}}=-\frac{\lambda}{m}$ becomes constant. To keep $\lambda$ small, it is indispensable to minimize the contact segment mass and to align the inertia main axis perpendicular to the impact direction - requirements that seek for an additional foot element.

\section{Passive Compliant Foot Segment}

Inspired by biomechanical studies (e.g.[9]) that pointed out multiple advantages of 3-segmented legs, we developed the foot element depicted in Figure 
1. This segment is built as lightweight as possible, since the contact inertia $\boldsymbol{\Lambda}_{s}$ is proportional to the mass of the most distal segment. Hence, the structurally optimized foot was manufactured as a single high-tensile aluminum part that has a total weight of only $125 \mathrm{~g}$ including a high resolution joint encoder (80'000 qc/rev), 10 digital distributed ground contact switches, and an orthopedic foot sole. In the current setup, the two modular ankle springs weight $56 \mathrm{~g}$ and produce a rotational stiffness of $c_{a}=10 \mathrm{Nm} / \mathrm{rad}$.

Previous studies conducted in our group aimed at finding optimal periodic gaits for a given robot model [3]. Periodicity in this example means that the system defined through parameters $\mathbf{p}$ passes through the same states $\mathbf{x}$ at every apex transit (highest position in flight phase), respectively that the stride-to-stride transfer function $P$ maps the periodic states onto themselves $\mathbf{x}^{k+1}=P^{*}\left(\mathbf{x}^{k}, \mathbf{p}, \mathbf{u}\right)=\mathbf{x}^{*}$. In the present work, we extended this and simultaneously optimized the actuator input $\mathbf{u}(t)$ and ankle joint stiffness $c_{a}$ by minimizing the positive mechanical actuator work (joint torque $\boldsymbol{\tau}$ )

$$
\underset{c_{a}, \mathbf{u}(t)}{\operatorname{minimize}} \quad f\left(\mathbf{x}^{*}, \mathbf{p}, \mathbf{u}(t)\right)=\int_{0}^{T_{h o p}} \max (0, \boldsymbol{\tau} \dot{\mathbf{u}}) d t
$$

subject to the motion periodicity and hopping height constraints

$$
\begin{aligned}
P^{*}\left(\mathbf{x}^{*}, \mathbf{p}, \mathbf{u}(t)\right)-\mathbf{x}^{*} & =0 \\
\max (y(t))-h & =0 .
\end{aligned}
$$

In this optimization problem, we fixed the period time of motion $T_{h o p}$, the hopping height $h$, as well as all mechanical parameters $\mathbf{p}$ except the ankle joint stiffness $c_{a}$. The motor actuation $\mathbf{u}(t)$ is described as a time based Fourier series with 3 coefficients:

$$
\mathbf{u}(t)=\sum_{i=0}^{3} a_{i} \sin \left(i \frac{2 \pi}{T} t\right)+b_{i} \cos \left(i \frac{2 \pi}{T} t\right)
$$

This has the benefit that we can account for the motor bandwidth limitations by keeping the number of Fourier coefficients small. The solution of the optimization problem is depicted in Figure 3(a): The bars indicate the value of the cost function (positive actuator work), the solid line the impact loss of the 3 -segmented leg in comparison to the 2-segmented leg (dashed line). The actuator work as well as the total impact loss is reduced by increasing the stiffness. Up to about $10 \mathrm{Nm} / \mathrm{rad}$ the ankle spring is compliant enough to allow for a double contact phase (heel makes contact), above that, only the toe touches the ground. The obtained results are close to the theoretical predictions [10] for a massless leg which showed that $c_{\text {ankle }}=\frac{l_{\text {foot }}}{l_{\text {thigh }}} c_{k n e e}$ is required for a symmetric bending (Figure 3(b)). 


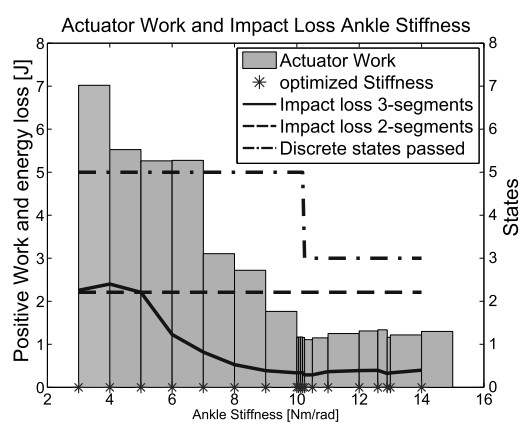

(a) ankle stiffness optimization

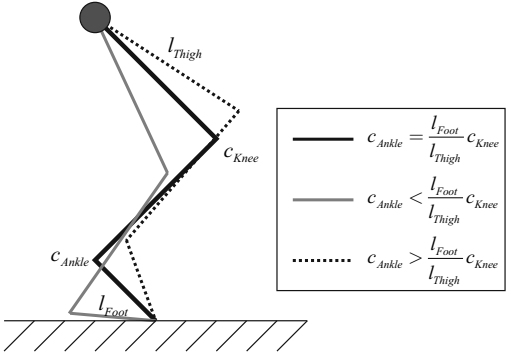

(b) massless 3-segmented leg

Fig. 3. The positive actuator work minimization (6) shows minimal energy consumption for a stiffness close to the point where heel strikes ground. (b) The ankle stiffness of a massless 3-segmented leg structure has a theoretical optimum [10].

These results indicate that in terms of efficiency, there is no benefit to make the ankle so stiff that heelstrike is fully avoided. However, considering controllability of the system, double contact is quite advantagous: since the ankle is not actuated, proper torque control (e.g., using virtual model control strategies [4]) is only applicable during this phase. In the time between toe and heel contact, a considerable amount of energy is stored in the ankle springs and the vertical heel speed is reduced such that the second impact at the heel is comparably small. In summary, the stiffness should be chosen to be just below the critical stiffness.

\section{Experimental Validation}

A simplified control structure was implemented in the 3-segmented leg to experimentally test the leg behavior in single legged running experiments for a varying ankle joint stiffness. The application of a passive compliant foot shows a big improvement of motor energy consumption (Figure $4(\mathrm{a}))$ : a compliant ankle spring $(4.0 \mathrm{Nm} / \mathrm{rad}$ (red-dotted), $2.85 \mathrm{~J})$ requires more energy compared to the stiffer springs $(7.2 \mathrm{Nm} / \mathrm{rad}$ (blue-solid), $10.5 \mathrm{Nm} / \mathrm{rad}$ (black-dashed), both $2.45 \mathrm{~J}$ ). Evaluating the hopping height (Figure 4(b)) shows the same tendency with a much higher hopping height $(0.175,0.185 \mathrm{~m})$ for the stiffer springs and a huge gap to the more compliant setup with a hopping height of only $0.04 \mathrm{~m}$. Hence, the optimization results could be entirely approved: as long as the heel still touches the ground, increasing the stiffness results in a remarkable improvement. As soon as the critical stiffness (only toe touches the ground) is reached, making the ankle stiffer does not improve the overall performance. 


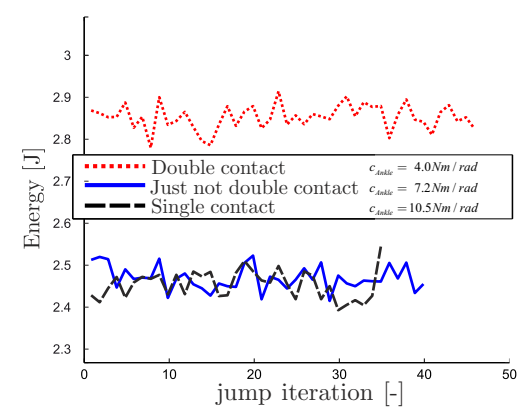

(a) motor energy consumption

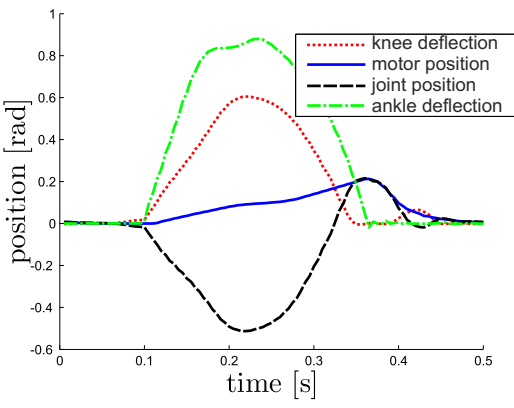

(c) position distribution

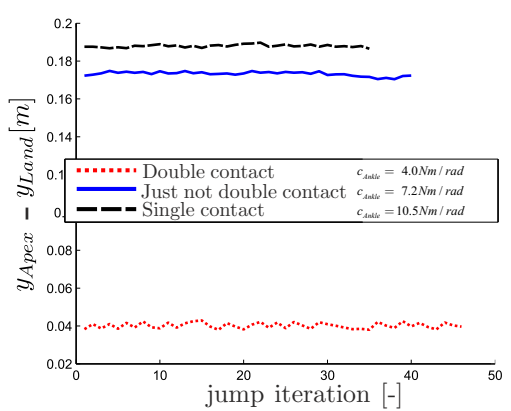

(b) jumping height

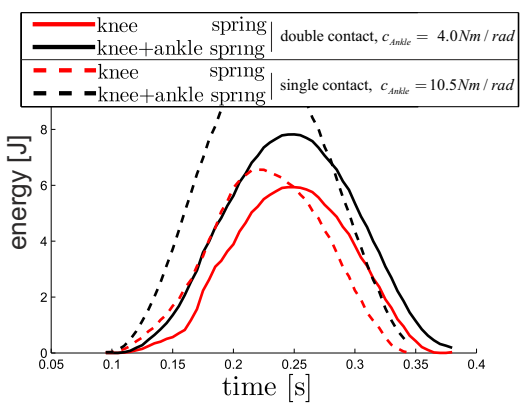

(d) energy distribution

Fig. 4. The foot segment was tested in single legged running experiments using a varying ankle joint stiffness.

The hopping experiment with an ankle stiffness of $10 \mathrm{Nm} / \mathrm{rad}$ (Figure $4(\mathrm{c})$ ) shows that the proposed control strategy provides only positive actuator power (joint velocity $>0$ during the entire stance phase) and the actual motor travel distance is only about $20 \%$ of the actual joint deflection.

The energetic improvement can be entirely assigned to the ankle. Comparing the stiffest and the most compliant ankle joint shows, that the energy stored in the knee remains the same while the total energy stored in the ankle and knee spring can be drastically increased (Figure 4(d)).

\section{Conclusion}

In the presented paper we showed theoretically as well as experimentally that the efficiency in single legged running with a 2-segmented leg is mechanically limited to a large extent due to energy losses by impacts at landing. As a biologically inspired solution, a highly optimized foot segment with joint torque and contact sensing capabilities was developed. A 
parameter and actuator optimization framework allowed to choose the best ankle joint stiffness and actuation input for highly efficient planar running. Due to the lower impacts at landing, the new flight configuration is less crouched than with the 2-segmented leg. A thorough experimental evaluation in planar running confirmed the theoretical expectations.

Appendix A. Proof of Kinetic Energy Loss

$$
\begin{aligned}
& \Delta E_{k i n} \quad=\quad \frac{1}{2} \dot{\mathbf{q}}^{+T} \mathbf{M} \dot{\mathbf{q}}^{+}-\frac{1}{2} \dot{\mathbf{q}}^{-T} \mathbf{M} \dot{\mathbf{q}}^{-}=\frac{1}{2}\left(\dot{\mathbf{q}}^{+}+\dot{\mathbf{q}}^{-}\right) \mathbf{M}\left(\dot{\mathbf{q}}^{+}-\dot{\mathbf{q}}^{-}\right) \\
& \dot{\mathbf{q}}^{+}=\dot{\mathbf{q}}^{-}+\Delta \dot{\mathbf{q}} \quad \frac{1}{2}\left(\Delta \dot{\mathbf{q}}+2 \dot{\mathbf{q}}^{-}\right) \mathbf{M} \Delta \dot{\mathbf{q}} \\
& \Delta \dot{\mathbf{q}}=-\overline{\overline{\mathbf{J}}}_{s} \mathbf{J}_{s} \dot{\mathbf{q}}^{-} \quad-\dot{\mathbf{q}}^{-T} \mathbf{M} \overline{\mathbf{J}}_{s} \mathbf{J}_{s} \dot{\mathbf{q}}^{-}+\frac{1}{2} \Delta \dot{\mathbf{q}}^{T} \mathbf{M} \Delta \dot{\mathbf{q}} \\
& \mathbf{J}_{s} \underline{\underline{\mathbf{J}_{s}}}=\mathbf{I} \quad-\dot{\mathbf{q}}^{-T} \mathbf{M} \overline{\mathbf{J}}_{s} \mathbf{J}_{s} \overline{\mathbf{J}}_{s} \mathbf{J}_{s} \dot{\mathbf{q}}^{-}+\frac{1}{2} \Delta \dot{\mathbf{q}}^{T} \mathbf{M} \Delta \dot{\mathbf{q}}
\end{aligned}
$$

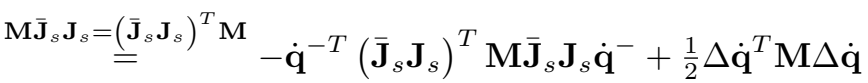

$$
\begin{aligned}
& \overline{\mathbf{J}}_{s} \mathbf{J}_{s} \dot{\dot{\mathbf{q}}}^{-}=\Delta \dot{\mathbf{q}} \quad-\frac{1}{2} \Delta \dot{\mathbf{q}}^{T} \mathbf{M} \Delta \dot{\mathbf{q}} \\
& \Delta \dot{\mathbf{q}}=\mathbf{M}^{-1} \mathbf{J}_{s} \boldsymbol{\Lambda}_{s} \Delta \dot{\mathbf{r}}_{s}-\frac{1}{2} \Delta \dot{\mathbf{r}}_{s}^{T} \boldsymbol{\Lambda}_{s} \underbrace{\mathbf{J}_{s} \mathbf{M}^{-1} \mathbf{M M}^{-1} \mathbf{J}_{s}^{T}}_{\boldsymbol{\Lambda}_{s}^{-1}} \boldsymbol{\Lambda}_{s} \Delta \dot{\mathbf{r}}_{s} \\
& =\quad-\frac{1}{2} \Delta \dot{\mathbf{r}}_{s}^{T} \boldsymbol{\Lambda}_{s} \Delta \dot{\mathbf{r}}_{s}
\end{aligned}
$$

\section{References}

1. C. T. Farley, J. Glasheen and T. A. Mcmahon, Journal of Experimental Biology 185, 71 (1993).

2. M. Hutter, C. D. Remy, M. H. Hoepflinger and R. Siegwart, High compliant series elastic actuation for the robotic leg scarleth, in CLAWAR, 2011.

3. C. D. Remy, Optimal expoitation of natrual dynamics in legged locomotion, $\mathrm{PhD}$ thesis2011.

4. M. Hutter, C. D. Remy, M. H. Hoepflinger and R. Siegwart, Scarleth: Design and control of a planar running robot, in IROS, 2011.

5. J. G. D. Karssen, M. Haberland, M. Wisse and S. Kim, The optimal swing-leg retraction rate for running, in ICRA, 2011.

6. O. Khatib, IEEE Journal of Robotics and Automation 3, 43 (1987).

7. J. P. Schmiedeler and K. J. Waldron, Advances in Robot Kinematics, 129 (2000).

8. H. Asada, Journal of Dynamic Systems Measurement and ControlTransactions of the Asme 105 (1983).

9. R. Blickhan, A. Seyfarth, H. Geyer, S. Grimmer, H. Wagner and M. Guenther, Philosophical Transactions of the Royal Society 365, 199 (2007).

10. A. Seyfarth, M. Guenther and R. Blickhan, Biological Cybernetics 84, 365 (2001). 